\title{
The Hopf boundary point lemma for vector bundle sections
}

\author{
Artem Pulemotov
}

\begin{abstract}
The paper establishes a version of the Hopf boundary point lemma for sections of a vector bundle over a manifold with boundary. This result may be viewed as a counterpart to the tensor maximum principle obtained by R. Hamilton in 1986. Potential applications include the study of various geometric flows and the construction of invariant sets for geometric boundary value problems.
\end{abstract}

Mathematics Subject Classification (2000). 35B50, 53C44, 35K50.

Keywords. Heat equation, maximum principle, Hopf lemma, parabolic system.

\section{Introduction}

The present paper concerns the solutions of a second-order partial differential equation in a vector bundle over a manifold with boundary. Let us describe the rootage of the considered problem.

The maximum principle for sections of a general vector bundle over a closed manifold was originally obtained in [9]. This statement is also known as the tensor maximum principle. It proved to be a powerful implement in the study of the Ricci flow; see [5]. In particular, it was used to establish important facts about fourmanifolds with nonnegative curvature operator. Other applications were considered, as well; see, for instance, [3], [10], and [2]. In particular, the paper [10] establishes the important matrix Li-Yau inequality for solutions of the heat equation.

A specific version of the maximum principle for sections appeared in [8]. This version only applied to 2-tensors. Several generalizations of the maximum principle for sections can be found in [6]. We refer to [4] and Chapter 4 of [5] for an overview of relevant results. Once again, we emphasize that the theory discussed above has been developed on closed manifolds.

The maximum principle for sections may be regarded as an evolution of the maximum principle for systems of scalar parabolic equations obtained in [16]. It must be noted that the statement in [16] has become a powerful implement in the study of parabolic systems. In particular, it was applied to the investigation of the 
existence and the asymptotic behavior of solutions. We refer to Chapter 14 of [15] for several relevant results and a vast bibliography; some of the references not mentioned there are [13], [7], [12], and [1].

An important comment should be made at this point. The maximum principles discussed above rely on the concept of an invariant set. The definition of an invariant set for a system of scalar parabolic equations can be found, for example, in Chapter 14 of [15]. This definition generalizes easily to cover the case of an equation for vector bundle sections. We remark that invariant sets should be viewed from a slightly different standpoint when the boundary conditions are specified for the solutions; see, for instance, [13], [11], and [12].

The paper [14], being devoted to the study of the Ricci flow on manifolds with boundary, offers a specific version of the Hopf boundary point lemma. This version applies to 2-tensors over a manifold with boundary. In essence, it is an analogue of the maximum principle for 2-tensors proved in [8]. At the same time, in spite of the fact that the universal maximum principle for sections suggested in [9] is a recognized powerful tool, no counterparts of this statement have yet been obtained in the presence of a boundary. Section 2 of the present paper establishes a general version of the Hopf boundary point lemma. Our statement applies to sections of a general vector bundle over a manifold with boundary. It appears to constitute a comprehensive counterpart to the maximum principle suggested in [9].

After proving our Hopf lemma for sections, we state three of its immediate corollaries. They are all closely related to the concept of an invariant set. The first corollary may be viewed as the basic maximum principle for sections of a vector bundle over a manifold with boundary. The second corollary shows that the maximum principle of [9] holds in the presence of a boundary provided that Neumann type boundary conditions are imposed on the sections in question. Such a result is expected to prove useful in the study of the Ricci flow; cf. [14]. The third corollary provides an explicit connection between invariant sets of an equation for vector bundle sections and the boundary conditions specified for the solutions. In certain situations, it allows to find an invariant set for a given boundary value problem. (In one form or another, this task was addressed in many works; see, for instance, [11], Chapter 14 of [15], [12], and [1].) Alternatively, the third corollary may be used to construct a relatively sophisticated boundary value problem with a given invariant set.

Section 3 of the present paper explains how our Hopf lemma for sections applies to a system of scalar parabolic equations similar to the one studied in [16]. Roughly speaking, we reformulate our statement for sections of a trivial bundle equipped with the standard connection. An analogous, although not exactly the same, result for parabolic systems can be found in [13]. We should note that the specific framework of Section 3 enables us to refine the smoothness assumptions imposed in Section 2. 


\section{The Hopf lemma for sections}

Consider a smooth, compact, connected, oriented Riemannian manifold $M$ with (possibly empty) boundary $\partial M$. We use the notation $v(x)$ for the outward unit normal to $\partial M$ at the point $x \in \partial M$. Let $V$ be a vector bundle over $M$. The fiber of $V$ over $x \in M$ will be denoted by $V_{x}$. The designation $\pi(v)$ refers to the projection of $v \in V$ onto $M$. We suppose $V$ is equipped with a fiber metric $\langle\cdot, \cdot\rangle$. Let $\|\cdot\|$ stand for the corresponding norm.

Consider a time-dependent section $f(t, x)$ of the vector bundle $V$. In what follows, the time parameter $t$ varies through the interval $[0, T]$ with a fixed $T>0$. Choose a connection $A$ in $V$ compatible with $\langle\cdot, \cdot\rangle$. Let $\nabla f(t, x)$ denote the covariant derivative of $f(t, x)$ with respect to $A$. We write $\nabla_{\chi} f(t, x)$ to indicate the application of $\nabla f(t, x)$ to the tangent vector $\chi \in T_{x} M$. Employing the connection $A$ in $V$ and the Levi-Civita connection in the cotangent bundle $T^{*} M$, one can define the second covariant derivative $\nabla^{2} f(t, x)$. We write $\nabla_{\chi_{1}, \chi_{2}}^{2} f(t, x)$ to indicate the application of $\nabla^{2} f(t, x)$ to the vectors $\chi_{1}, \chi_{2} \in T_{x} M$. The Laplacian $\Delta$ acts on the section $f(t, x)$ by taking the trace of $\nabla^{2} f(t, x)$. We refer to Chapter 4 of [5] for the details of defining the Laplacian. ${ }^{1}$

Let $\phi(t, v)$ be a time-dependent mapping of $V$ into itself such that $\phi(t, v) \in V_{\pi(v)}$ for any $(t, v) \in[0, T] \times V$. Suppose every compact set $U \subset V$ admits a constant $C_{\phi}(U)>0$ satisfying

$$
\left\|\phi\left(t, v_{1}\right)-\phi\left(t, v_{2}\right)\right\| \leq C_{\phi}(U)\left\|v_{1}-v_{2}\right\| .
$$

The estimate must hold for any $t \in(0, T)$, and any $v_{1}, v_{2} \in U$ subject to $\pi\left(v_{1}\right)=$ $\pi\left(v_{2}\right)$. Let $\zeta(t, x)$ be a time-dependent vector field on $M$. Suppose $f(t, x)$ solves the second-order equation

$$
\frac{\partial}{\partial t} f(t, x)=\Delta f(t, x)+\nabla_{\zeta(t, x)} f(t, x)+\phi(t, f(t, x))
$$

on $(0, T) \times M$. In particular, $f(t, x)$ must be continuous in $t \in[0, T]$ and $C^{1}$ differentiable in $t \in(0, T)$.

Consider a nonempty set $W \subset V$. We assume $W$ is invariant under the parallel translation with respect to the connection $A$ fixed in $V$. The set $W_{x}=W \cap V_{x}$ must be closed and convex in the fiber $V_{x}$ for every $x \in M$. When writing $\partial W_{x}$, we refer to the boundary of $W_{x}$ in $V_{x}$. It should be noted that $\partial W_{x}$ is not required to be smooth for any $x \in M$. Given a point $\omega \in W$ subject to $\omega \in \partial W_{\pi(\omega)}$, we call $\lambda \in V_{\pi(\omega)}$ a supporting vector for $W$ at $\omega$ if $\|\lambda\|=1$ and the inequality $\langle\lambda, \sigma\rangle \leq\langle\lambda, \omega\rangle$ holds for all $\sigma \in W_{\pi(\omega)}$. The set of all the supporting vectors for $W$ at $\omega$ will be denoted by $S_{\omega} W$. In a sense, the elements of $S_{\omega} W$ are outward unit normals to $\partial W_{\pi(\omega)}$ at $\omega$.

\footnotetext{
${ }^{1}$ The denotations $\hat{\nabla}(t)(\bar{\nabla}(t) \varphi)(t, x)$ and $\hat{\Delta}$ are used in Chapter 4 of [5] for the objects denoted by $\nabla^{2} f(t, x)$ and $\Delta$ in the present paper.
} 
Introduce the notation

$$
\operatorname{dist}_{W} v=\inf _{\omega \in W_{\pi(v)}}\|v-\omega\|
$$

for $v \in V$. Let $\omega(v)$ be the unique point in $W_{\pi(v)}$ such that dist $W=\|v-\omega(v)\|$. Obviously, $\operatorname{dist}_{W} v$ represents the distance between $v \in V$ and $W_{\pi(v)}$, while $\omega(v)$ is the unique point in $W_{\pi(v)}$ closest to $v$. We call $(t, x) \in[0, T] \times M$ a maximal distance pair if

$$
\operatorname{dist}_{W} f(t, x)=\sup _{y \in M} \operatorname{dist}_{W} f(t, y)>0 .
$$

Let $\lambda(v)$ denote the difference $v-\omega(v)$ for $v \in V$.

We are now ready to formulate our Hopf lemma for sections. It should be remarked that the assumption on the mapping $\phi(t, v)$ in our statement is quite standard. Roughly speaking, we demand that $\phi(t, v)$ point into $W$ when $v$ is subject to $v \in \partial W_{\pi(v)}$. This is equivalent to the "ordinary differential equation assumption" employed in [9]; see Lemma 4.1 in [9].

Theorem 2.1. Suppose the solution $f(t, x)$ of equation (2.2) and the mapping $\phi(t, v)$ appearing in the right-hand side of equation (2.2) meet the following requirements:

(1) The initial value $f(0, x)$ lies in $W$ for all $x \in M$.

(2) The estimate $\langle\lambda, \phi(t, \omega)\rangle \leq 0$ holds for any $t \in(0, T)$, any $\omega \in W$ subject to $\omega \in \partial W_{\pi(\omega)}$, and any supporting vector $\lambda \in S_{\omega} W$.

If the value $f(t, x)$ lies outside of $W$ for some $(t, x) \in(0, T] \times M$, then there exists a maximal distance pair $\left(t_{\mathrm{pos}}, x_{\mathrm{pos}}\right) \in(0, T) \times \partial M$ such that the formula

$$
\left\langle\lambda\left(f\left(t_{\mathrm{pos}}, x_{\mathrm{pos}}\right)\right), \nabla_{\nu\left(x_{\mathrm{pos}}\right)} f\left(t_{\mathrm{pos}}, x_{\mathrm{pos}}\right)\right\rangle>0
$$

holds true.

Before proving the theorem, we need to make some preliminary arrangements. Given a real-valued function $\theta(t)$ on $[0, T)$, define

$$
\dot{\theta}^{+}(t)=\limsup _{h \rightarrow 0+} \frac{\theta(t+h)-\theta(t)}{h}
$$

for $t \in[0, T)$. The following lemma will be required; cf. Lemma 3.1 and Corollary 3.3 in [9], or Lemma 7 in [6].

Lemma 2.1. Suppose $\theta(t)$ is a non-negative continuous function on $[0, T)$ with $\theta(0)=0$. Suppose also $\theta(t)$ is not identically 0 on $[0, T)$. Given a constant $C>0$, there exists a point $t_{C} \in(0, T)$ such that $\dot{\theta}^{+}\left(t_{C}\right)>C \theta\left(t_{C}\right)$ and $\theta\left(t_{C}\right)>0$. 
Proof. Assume the existence of $C>0$ satisfying the estimate $\dot{\theta}^{+}(t) \leq C \theta(t)$ whenever $\theta(t)>0$. Introduce a new non-negative continuous function $\eta(t)=e^{-C t} \theta(t)$. Clearly, the equality $\eta(0)=0$ holds, and $\dot{\eta}^{+}(t) \leq 0$ whenever $\eta(t)>0$.

Fix $\epsilon_{1}, \epsilon_{2}>0$. We will now prove that $\eta(t) \leq \epsilon_{1} t+\epsilon_{2}$ for all $t \in[0, T)$. Let $a$ be the largest possible number in $(0, T]$ such that the inequality $\eta(t) \leq \epsilon_{1} t+\epsilon_{2}$ holds on $[0, a)$. (Since $\eta(0)=0<\epsilon_{2}$, the set of such numbers is not empty, and $a$ is well defined.) We claim that $a=T$. Indeed, if $a<T$, then $\eta(a)=\epsilon_{1} a+\epsilon_{2}>0$ by continuity and

$$
\limsup _{h \rightarrow 0+} \frac{\eta(a+h)-\eta(a)}{h} \leq 0
$$

But this implies $\eta(t) \leq \epsilon_{1} t+\epsilon_{2}$ on $[0, a+\delta)$ for some $\delta>0$, which contradicts the definition of $a$.

Thus $\eta(t) \leq \epsilon_{1} t+\epsilon_{2}$ for all $t \in[0, T)$. Since this inequality holds for any $\epsilon_{1}, \epsilon_{2}>0$, we can conclude that $\eta(t)$ is identically 0 . Hence $\theta(t)$ is identically 0 , which contradicts the suppositions of the lemma.

Proof of Theorem 2.1. It suffices to carry out the proof assuming $W$ is compact. In order to justify this statement, fix a number $R>0$ large enough to ensure that $\|f(t, x)\|<R$ and $\|\omega(f(t, x))\|<R$ for any $(t, x) \in[0, T] \times M$. Introduce the set $\widehat{W}=\{w \in W \mid\|w\| \leq R\}$. One can verify that $\widehat{W}$ is compact. Clearly, it is invariant under the parallel translation with respect to $A$, and its intersection with the fiber $V_{x}$ is closed and convex in $V_{x}$ for every $x \in M$. Let $\kappa(v)$ be a smooth function acting from $V$ to the interval $[0,1]$. We choose $\kappa(v)$ demanding that $\kappa(v)=1$ when $\|v\| \leq R$ and $\kappa(v)=0$ when $\|v\| \geq 2 R$. Define the time-dependent mapping $\hat{\phi}(t, v)$ of $V$ into itself by the formula $\hat{\phi}(t, v)=\kappa(v) \phi(t, v)$. Estimate (2.1) is obviously satisfied for $\hat{\phi}(t, v)$ with the constant $C_{\hat{\phi}}(U)=C_{\phi}(U)$ when the compact set $U$ is equal to $f([0, T] \times M) \cup \widehat{W}$. (We note that the proof of the theorem will not require estimate (2.1) to hold when $U$ is other than $f([0, T] \times M) \cup W$.) The section $f(t, x)$ would remain a solution of equation (2.2) if the mapping $\hat{\phi}(t, v)$ appeared in the righthand side of this equation instead of the mapping $\phi(t, v)$. A straightforward argument demonstrates that it suffices to prove the theorem with $W$ and $\phi(t, v)$ replaced by $\widehat{W}$ and $\hat{\phi}(t, v)$. Therefore, supposing $W$ is compact does not lead to a loss of generality.

Introduce the function

$$
s(t)=\sup _{x \in M} \operatorname{dist}_{W} f(t, x)
$$

for $t \in[0, T]$. Evidently, it is non-negative. One can show that $s(t)$ is continuous. Our requirement (1) implies that $s(0)=0$. If $f(t, x)$ lies outside of $W$ for some $(t, x) \in(0, T] \times M$, then $s(t)$ is not identically 0 on $[0, T]$. Assuming the assertion of the theorem fails to hold, we will prove the estimate $\dot{s}^{+}(t) \leq C s(t)$ for a fixed 
constant $C>0$ and an arbitrary $t \in(0, T)$ such that $s(t)>0$. Lemma 2.1 would then provide a contradiction.

Fix a point $t \in(0, T)$ satisfying $s(t)>0$. If $x \in M$ is subject to dist $W f(t, x)>0$, the equality

$$
\operatorname{dist}_{W} f(t, x)=\sup _{\omega \in \partial W_{x}} \sup _{\lambda \in S_{\omega} W}\langle\lambda, f(t, x)-\omega\rangle
$$

holds true. This implies

$$
\begin{aligned}
s(t) & =\sup _{(\omega, \lambda) \in \Omega}\langle\lambda, f(t, \pi(\omega))-\omega\rangle, \\
\Omega & =\left\{(\omega, \lambda) \in V \times V \mid \omega \in \partial W_{\pi(\omega)}, \lambda \in S_{\omega} W\right\} .
\end{aligned}
$$

The set $\Omega$ is compact in $V \times V$. Therefore, we can apply Lemma 9 in [6], see also Lemma 3.5 in [9], to conclude

$$
\begin{aligned}
\dot{s}^{+}(t) & \leq\left.\sup _{(\omega, \lambda) \in \Omega^{\prime}} \frac{\partial}{\partial r}\langle\lambda, f(r, \pi(\omega))-\omega\rangle\right|_{r=t}, \\
\Omega^{\prime} & =\{(\omega, \lambda) \in \Omega \mid s(t)=\langle\lambda, f(t, \pi(\omega))-\omega\rangle\} .
\end{aligned}
$$

Fix a pair $(\omega, \lambda) \in \Omega^{\prime}$. For brevity, we write $x$ instead of $\pi(\omega)$. The point $x \in M$ is thus fixed from now on. Assuming the assertion of the theorem fails to hold, we will show that $\left.\frac{\partial}{\partial r}\langle\lambda, f(r, x)-\omega\rangle\right|_{r=t} \leq C s(t)$ for a constant $C>0$ independent of $t$. This would yield the desired estimate $\dot{s}^{+}(t) \leq C s(t)$.

Equation (2.2) yields

$$
\begin{aligned}
\frac{\partial}{\partial r}\langle\lambda, & f(r, x)-\omega\rangle\left.\right|_{r=t} \\
& =\langle\lambda, \Delta f(t, x)\rangle+\left\langle\lambda, \nabla_{\zeta(t, x)} f(t, x)\right\rangle+\langle\lambda, \phi(t, f(t, x))\rangle .
\end{aligned}
$$

The inclusion $(\omega, \lambda) \in \Omega^{\prime}$ implies that $(t, x)$ is a maximal distance pair and the vector $\lambda$ coincides with $\frac{\lambda(f(t, x))}{\|\lambda(f(t, x))\|}$. If the assertion of the theorem were incorrect, then either $x$ would be in the interior of $M$ or $\left\langle\lambda, \nabla_{v(x)} f(t, x)\right\rangle$ would be non-positive. Assuming this alternative, we will estimate each of the three terms in the right-hand side of equation (2.4).

Let us establish the equality $\left\langle\lambda, \nabla_{\chi} f(t, x)\right\rangle=0$ for an arbitrary $\chi \in T_{x} M$. Obviously, it would imply

$$
\left\langle\lambda, \nabla_{\zeta(t, x)} f(t, x)\right\rangle=0 .
$$

At the first step, we consider a vector $\chi \in T_{x} M$ admitting a geodesic segment $\gamma_{\chi}(u)$ defined for $u \in\left[0, \epsilon_{\chi}\right]$ in such a way that $\gamma_{\chi}(0)=x$ and $\frac{d \gamma_{\chi}}{d u}(0)=\chi$. The number $\epsilon_{\chi}$ 
should be chosen small enough to ensure the geodesic segment's not intersecting itself. The initial goal is to show that $\left\langle\lambda, \nabla_{\chi} f(t, x)\right\rangle \leq 0$.

For the sake of brevity, we write $\gamma(u)$ instead of $\gamma_{\chi}(u)$ and $\epsilon$ instead of $\epsilon_{\chi}$. One can extend the vectors $\lambda$ and $\omega$ to parallel (with respect to the connection $A$ ) sections $\lambda^{\prime}(\gamma(u))$ and $\omega^{\prime}(\gamma(u))$ of the bundle $V$ defined along $\gamma(u)$. The covariant derivatives of $\lambda^{\prime}(\gamma(u))$ and $\omega^{\prime}(\gamma(u))$ with respect to $A$ at the point $x=\gamma(0)$ exist in the direction of $\chi$. Writing $\nabla_{\chi} \lambda^{\prime}(x)$ and $\nabla_{\chi} \omega^{\prime}(x)$ for these covariant derivatives, we can easily see that $\nabla_{\chi} \lambda^{\prime}(x)=0$ and $\nabla_{\chi} \omega^{\prime}(x)=0$.

Introduce the function $g(u)=\left\langle\lambda^{\prime}(\gamma(u)), f(t, \gamma(u))-\omega^{\prime}(\gamma(u))\right\rangle$ on $[0, \epsilon]$. Obviously, $g(0)=s(t)$. Using the fact that the parallel transport is an isometry of the fibers, one proves $\omega^{\prime}(\gamma(u)) \in \partial W_{\gamma(u)}$ and $\lambda^{\prime}(\gamma(u)) \in S_{\omega^{\prime}(\gamma(u))} W$ for any $u \in[0, \epsilon]$. These inclusions imply the inequality

$$
g(0)=s(t) \geq\left\langle\lambda^{\prime}(\gamma(u)), f(t, \gamma(u))-\omega^{\prime}(\gamma(u))\right\rangle=g(u)
$$

for any $u \in[0, \epsilon]$. As a consequence, the function $g(u)$ has a maximum at 0 , and the one-sided derivative $\frac{d g}{d u}(0)$ is non-positive. Since the connection $A$ is compatible with the fiber metric, we have the formula

$$
\begin{aligned}
\left\langle\lambda, \nabla_{\chi} f(t, x)\right\rangle & =\left\langle\nabla_{\chi} \lambda^{\prime}(x), f(t, x)\right\rangle+\left\langle\lambda, \nabla_{\chi} f(t, x)\right\rangle \\
& =\left.\frac{\partial}{\partial u}\left\langle\lambda^{\prime}(\gamma(u)), f(t, \gamma(u))\right\rangle\right|_{u=0}=\frac{d g}{d u}(0) .
\end{aligned}
$$

Hence $\left\langle\lambda, \nabla_{\chi} f(t, x)\right\rangle \leq 0$.

Choose an orthonormal basis $\left\{e_{1}, \ldots, e_{n}\right\}$ of the tangent space $T_{x} M$. We will show that $\left\langle\lambda, \nabla_{e_{k}} f(t, x)\right\rangle=0$ for any $k=1, \ldots, n$. Suppose $x$ lies in the interior of $M$. Then a geodesic segment $\gamma_{e_{k}}(u)$, the parameter $u$ varying through $\left[0, \epsilon_{e_{k}}\right]$, subject to $\gamma_{e_{k}}(0)=x$ and $\frac{d \gamma_{e_{k}}}{d u}(0)=e_{k}$ exists for any $k=1, \ldots, n$. As a consequence, the scalar products $\left\langle\lambda, \nabla_{e_{k}} f(t, x)\right\rangle$ are non-positive. Substituting $-e_{k}$ for $e_{k}$ and repeating the argument, we conclude that the scalar products $\left\langle\lambda, \nabla_{e_{k}} f(t, x)\right\rangle$ are also non-negative. Thus $\left\langle\lambda, \nabla_{e_{k}} f(t, x)\right\rangle=0$ for any $k=1, \ldots, n$.

Suppose $x$ lies in the boundary of $M$. Without loss of generality, we assume that $e_{n}$ coincides with the inward normal to the boundary of $M$. It is easy to verify the existence of a geodesic segment $\gamma_{e_{n}}(u)$ defined for $u \in\left[0, \epsilon_{e_{n}}\right]$ in such a way that $\gamma_{e_{n}}(0)=x$ and $\frac{d \gamma_{e_{n}}}{d u}(0)=e_{n}$. Consequently, the scalar product $\left\langle\lambda, \nabla_{e_{n}} f(t, x)\right\rangle$ is non-positive. At the same time, our hypothesis implies that $\left\langle\lambda, \nabla_{e_{n}} f(t, x)\right\rangle=$ $-\left\langle\lambda, \nabla_{v(x)} f(t, x)\right\rangle$ is non-negative. Thus $\left\langle\lambda, \nabla_{e_{n}} f(t, x)\right\rangle=0$. Provided $n \geq 2$, we now prove that $\left\langle\lambda, \nabla_{e_{k}} f(t, x)\right\rangle=0$ for $k=1, \ldots, n-1$. The situation is slightly more complicated here because a geodesic emanating from $x$ in the direction of $e_{k}$ does not necessarily exist. In order to overcome this problem, we will carry out an approximation procedure. Namely, fix a sequence $\left(e_{k}^{m}\right)_{m=1}^{\infty}$ converging to $e_{k}$ for every $k=1, \ldots, n-1$. We choose the vectors $e_{k}^{m}$ demanding that the scalar 
product of $e_{k}^{m}$ and $e_{n}$ with respect to the Riemannian metric in $M$ be strictly positive. Given $k$ and $m$, it is easy to verify the existence of a geodesic segment $\gamma_{e_{k}^{m}}(u)$, the parameter $u$ varying through $\left[0, \epsilon_{e_{k}^{m}}\right]$, subject to $\gamma_{e_{k}^{m}}(0)=x$ and $\frac{d \gamma_{e_{k}^{m}}}{d u}(0)=e_{k}^{m}$. As a consequence, $\left\langle\lambda, \nabla_{e_{k}^{m}} f(t, x)\right\rangle \leq 0$. The convergence of $\left(e_{k}^{m}\right)_{m=1}^{\infty}$ to $e_{k}$ then implies $\left\langle\lambda, \nabla_{e_{k}} f(t, x)\right\rangle \leq 0$. Substituting $-e_{k}$ for $e_{k}$ and repeating the argument, we conclude that $\left\langle\lambda, \nabla_{e_{k}} f(t, x)\right\rangle \geq 0$. Thus $\left\langle\lambda, \nabla_{e_{k}} f(t, x)\right\rangle=0$ for $k=1, \ldots, n-1$.

By virtue of the established equalities, $\left\langle\lambda, \nabla_{\chi} f(t, x)\right\rangle=0$ for an arbitrary $\chi \in$ $T_{x} M$. This clearly proves formula (2.5).

Our next goal is to obtain the estimate

$$
\langle\lambda, \Delta f(t, x)\rangle \leq 0
$$

As before, consider a vector $\chi \in T_{x} M$ admitting a geodesic segment $\gamma_{\chi}(u)$ defined for $u \in\left[0, \epsilon_{\chi}\right]$ in such a way that $\gamma_{\chi}(0)=x$ and $\frac{d \gamma_{\chi}}{d u}(0)=\chi$. The number $\epsilon_{\chi}$ should be small enough to ensure the absence of self-intersections. We now show that $\left\langle\lambda, \nabla_{\chi, \chi}^{2} f(t, x)\right\rangle \leq 0$. This would provide us with a basis for the proof of estimate (2.6).

Again, we write $\gamma(u)$ instead of $\gamma_{\chi}(u)$ and $\epsilon$ instead of $\epsilon_{\chi}$. It will be convenient to use the notation $\gamma^{\prime}(u)$ for $\frac{d \gamma}{d u}(u)$. A parallel section $\lambda^{\prime}(\gamma(u))$ of the bundle $V$ along $\gamma(u)$ has been introduced above. The covariant derivative of this section with respect to the connection $A$ at the point $\gamma(u)$ exists in the direction of $\gamma^{\prime}(u)$ for any $u \in[0, \epsilon)$. Writing $\nabla_{\gamma^{\prime}(u)} \lambda^{\prime}(\gamma(u))$ for this covariant derivative, we can easily see that $\nabla_{\gamma^{\prime}(u)} \lambda^{\prime}(\gamma(u))=0$ for any $u \in[0, \epsilon)$.

Since $A$ is compatible with the fiber metric, the equality

$$
\begin{aligned}
\left\langle\lambda, \nabla_{\chi, \chi}^{2} f(t, x)\right\rangle= & \left\langle\nabla_{\chi} \lambda^{\prime}(x), \nabla_{\chi} f(t, x)\right\rangle+\left\langle\lambda, \nabla_{\chi, \chi}^{2} f(t, x)\right\rangle \\
= & \left.\frac{\partial}{\partial u}\left\langle\lambda^{\prime}(\gamma(u)), \nabla_{\gamma^{\prime}(u)} f(t, \gamma(u))\right\rangle\right|_{u=0} \\
= & \frac{\partial}{\partial u}\left(\left\langle\nabla_{\gamma^{\prime}(u)} \lambda^{\prime}(\gamma(u)), f(t, \gamma(u))\right\rangle\right. \\
& \left.\quad+\left\langle\lambda^{\prime}(\gamma(u)), \nabla_{\gamma^{\prime}(u)} f(t, \gamma(u))\right\rangle\right)\left.\right|_{u=0} \\
= & \left.\frac{\partial^{2}}{\partial u^{2}}\left\langle\lambda^{\prime}(\gamma(u)), f(t, \gamma(u))\right\rangle\right|_{u=0} \\
= & \frac{d^{2} g}{d u^{2}}(0)
\end{aligned}
$$

holds true. The function $g(u)$ introduced above has a maximum at 0 . It has been proven that $\frac{d g}{d u}(0)=\left\langle\lambda, \nabla_{\chi} f(t, x)\right\rangle=0$. Therefore $\frac{d^{2} g}{d u^{2}}(0) \leq 0$, which yields $\left\langle\lambda, \nabla_{\chi, \chi}^{2} f(t, x)\right\rangle \leq 0$. 
Suppose $x$ lies in the interior of $M$. Then every vector from the chosen above basis $\left\{e_{1}, \ldots, e_{n}\right\}$ appears as a tangent vector for a certain geodesic segment emanating from $x$. As a consequence, $\left\langle\lambda, \nabla_{e_{k}, e_{k}}^{2} f(t, x)\right\rangle \leq 0$ for every $k=1, \ldots, n$.

Suppose $x$ lies in the boundary of $M$. Recall that $e_{n}$ is assumed to coincide with the inward normal to the boundary of $M$. As mentioned before, $e_{n}$ appears as a tangent vector for a certain geodesic segment emanating from $x$. Therefore, $\left\langle\lambda, \nabla_{e_{n}, e_{n}}^{2} f(t, x)\right\rangle \leq 0$. Provided $n \geq 2$, we can approximate the other basis vectors with the previously fixed sequences $\left(e_{k}^{m}\right)_{m=1}^{\infty}$ to conclude that $\left\langle\lambda, \nabla_{e_{k}, e_{k}}^{2} f(t, x)\right\rangle \leq 0$ for every $k=1, \ldots, n-1$.

According to the definition of the Laplacian,

$$
\langle\lambda, \Delta f(t, x)\rangle=\sum_{k=1}^{n}\left\langle\lambda, \nabla_{e_{k}, e_{k}}^{2} f(t, x)\right\rangle .
$$

By virtue of the established inequalities, all the terms in the right-hand side are nonpositive. This clearly implies formula (2.6).

Finally, let us prove the estimate

$$
\langle\lambda, \phi(t, f(t, x))\rangle \leq C s(t)
$$

with a constant $C>0$ independent of $t$. The vector $\lambda$ belongs to $S_{\omega} W$. It must also belong to $S_{\omega(f(t, x))} W$, although $\omega$ does not necessarily coincide with $\omega(f(t, x))$. (Recall that $\omega(f(t, x))$ stands for the unique point in $W_{x}$ closest to $f(t, x)$.) In accordance with our requirement (2), $\langle\lambda, \phi(t, \omega(f(t, x)))\rangle \leq 0$. Hence the estimate

$$
\begin{aligned}
\langle\lambda, \phi(t, f(t, x))\rangle & \leq\langle\lambda, \phi(t, f(t, x))\rangle-\langle\lambda, \phi(t, \omega(f(t, x)))\rangle \\
& \leq\|\phi(t, f(t, x))-\phi(t, \omega(f(t, x)))\| \\
& \leq C\|f(t, x)-\omega(f(t, x))\|=C s(t)
\end{aligned}
$$

holds with the constant $C>0$ equal to the constant $C_{\phi}(f([0, T] \times M) \cup W)>0$ given by formula (2.1). This concludes the proof of (2.7). Remark that the argument we used does not depend on whether $x$ is in the boundary of $M$ or in the interior of $M$.

Equation (2.4) now provides $\left.\frac{\partial}{\partial r}\langle\lambda, f(r, x)-\omega\rangle\right|_{r=t} \leq C s(t)$. As mentioned before, this inequality implies $\dot{s}^{+}(t) \leq C s(t)$, which is impossible in view of Lemma 2.1.

Remark 2.1. The assumption on the mapping $\phi(t, v)$ imposed by the theorem may be slightly refined. Namely, it suffices to demand that the estimate $\langle\lambda, \phi(t, \omega)\rangle \leq 0$ hold when $\omega$ is equal to $\omega(f(t, x))$ and $\lambda$ is equal to $\lambda(f(t, x))$ for every maximal distance pair $(t, x) \in(0, T) \times M$.

Remark 2.2. If the boundary of $M$ is empty, then the suppositions of the theorem cannot be satisfied simultaneously. In this case, requirements (1) and (2) ensure that 
$f(t, x)$ cannot lie outside of $W$. This fact is essentially equivalent to the maximum principle obtained in [9].

Remark 2.3. The theorem would prevail if the Riemannian metric in $M$ and the connection $A$ fixed in $V$ depended on the time parameter $t \in[0, T]$. Of course, then we would have to modify some of the assumptions imposed above. Firstly, the connection $A(t)$ fixed in $V$ at time $t$ would be required to be compatible with the fiber metric $\langle\cdot, \cdot\rangle$ for all $t \in(0, T)$. Secondly, the set $W$ would have to be invariant under the parallel translation with respect to $A(t)$ for all $t \in(0, T)$. The details of defining the Laplacian and writing down equation (2.2) in the situation under discussion can be found in Chapter 4 of [5]. The covariant derivative and the outward normal in formula (2.3) would have to be computed with respect to the connection $A\left(t_{\mathrm{pos}}\right)$ and the Riemannian metric in $M$ at time $t_{\text {pos }}$.

We will now formulate three immediate corollaries of Theorem 2.1. The following statement may be viewed as the basic maximum principle for sections of a vector bundle over a manifold with boundary.

Corollary 2.1. Suppose the solution $f(t, x)$ and the mapping $\phi(t, v)$ meet requirements (1) and (2) of Theorem 2.1. If $f(t, x)$ lies in $W$ for all $(t, x) \in(0, T) \times \partial M$, then $f(t, x)$ lies in $W$ for all $(t, x) \in[0, T] \times M$.

The following statement shows that the maximum principle of [9] holds for $f(t, x)$ provided that Neumann type boundary conditions are imposed.

Corollary 2.2. Suppose the solution $f(t, x)$ and the mapping $\phi(t, v)$ meet requirements (1) and (2) of Theorem 2.1. If the boundary condition

$$
\nabla_{\nu(x)} f(t, x)=0
$$

is satisfied for all $(t, x) \in(0, T) \times \partial M$, then $f(t, x)$ lies in $W$ for all $(t, x) \in$ $[0, T] \times M$.

Let $\bar{\lambda}(v)$ be a mapping of $V$ into itself such that $\bar{\lambda}(v) \in V_{\pi(v)}$ for any $v \in V$. The following statement establishes an explicit connection between invariant sets of equation (2.2) and the boundary conditions specified for the solutions.

Corollary 2.3. Suppose the solution $f(t, x)$ and the mapping $\phi(t, v)$ meet requirements (1) and (2) of Theorem 2.1. Suppose also $\bar{\lambda}(v)=\lambda(v)$ for any $v \in V$ lying outside of $W$. If the boundary condition

$$
\left\langle\bar{\lambda}(f(t, x)), \nabla_{v(x)} f(t, x)\right\rangle=0
$$

is satisfied for all $(t, x) \in(0, T) \times \partial M$, then $f(t, x)$ lies in $W$ for all $(t, x) \in$ $[0, T] \times M$. 
It should be noted that both Corollary 2.1 and Corollary 2.2 can be deduced from Corollary 2.3.

\section{Systems of parabolic equations}

We will now explain how Theorem 2.1 applies to a parabolic system similar to the one studied in [16]. We remark that dealing with a parabolic system rather than an equation for vector bundle sections enables us to refine the smoothness assumptions imposed in Section 2.

Let $M$ be the closure of a bounded domain in $\mathbb{R}^{n}$ with $C^{1}$-differentiable boundary $\partial M$. We use the designation $v(x)$ for the outward unit normal to $\partial M$ at the point $x \in \partial M$. Differentiation with respect to $x \in M$ in the direction of $v(x)$ will be denoted by $\frac{\partial}{\partial \nu}$. Let $x_{1}, \ldots, x_{n}$ be the standard coordinates in $\mathbb{R}^{n}$.

Consider a collection of time-dependent real-valued functions $f_{i}(t, x)$ on $M$ indexed by $i=1, \ldots, m$. The time parameter $t$ varies through the interval $[0, T]$. We write $f(t, x)$ for the vector $\left(f_{1}(t, x), \ldots, f_{m}(t, x)\right)$. Roughly speaking, $f(t, x)$ appears as a time-dependent section of the product bundle $M \times \mathbb{R}^{m}$.

Let $\phi_{i}(t, x, v)$ be a collection of time-dependent real-valued functions on $M \times \mathbb{R}^{m}$ indexed by $i=1, \ldots, m$. Again, $\phi(t, x, v)$ stands for the vector $\left(\phi_{1}(t, x, v), \ldots\right.$, $\left.\phi_{m}(t, x, v)\right)$. The denotations $\langle\cdot, \cdot\rangle$ and $\|\cdot\|$ refer to the standard Euclidean scalar product and the standard Euclidean norm in $\mathbb{R}^{m}$. We demand that every compact set $U \subset \mathbb{R}^{m}$ admit a constant $C_{\phi}(U)>0$ such that

$$
\left\|\phi\left(t, x, v_{1}\right)-\phi\left(t, x, v_{2}\right)\right\| \leq C_{\phi}(U)\left\|v_{1}-v_{2}\right\| .
$$

The estimate must hold for any $t \in(0, T)$, any $x \in M$, and any pair of vectors $v_{1}, v_{2} \in U$. Roughly speaking, $\phi(t, x, v)$ appears as a time-dependent mapping of the bundle $M \times \mathbb{R}^{m}$ into itself. The above inequality may then be viewed as a special case of inequality (2.1).

Fix time-dependent real-valued functions $\zeta_{j}(t, x)$ on $M$ for $j=1, \ldots, n$. We suppose $f_{i}(t, x)$ solves the second-order equation

$$
\frac{\partial}{\partial t} f_{i}(t, x)=\sum_{j=1}^{n} \frac{\partial^{2}}{\partial x_{j}^{2}} f_{i}(t, x)+\sum_{j=1}^{n} \zeta_{j}(t, x) \frac{\partial}{\partial x_{j}} f_{i}(t, x)+\phi_{i}(t, x, f(t, x))
$$

on $(0, T) \times M$ for every $i=1, \ldots, m$. In particular, $f_{i}(t, x)$ must be continuous in $t \in[0, T], C^{1}$-differentiable in $t \in(0, T)$, and $C^{2}$-differentiable in $x \in M$. The collection of equations (3.1) for $i=1, \ldots, m$ may be viewed as a special case of equation (2.2).

Consider a nonempty closed convex set $W \subset \mathbb{R}^{m}$. Given a point $\omega \in \partial W$, we call $\lambda \in \mathbb{R}^{m}$ a supporting vector for $W$ at $\omega$ if $\|\lambda\|=1$ and the inequality $\langle\lambda, \sigma\rangle \leq\langle\lambda, \omega\rangle$ 
holds for all $\sigma \in W$. Let $S_{\omega} W$ signify the set of all the supporting vectors for $W$ at $\omega$. We write $\operatorname{dist}_{W} v$ to denote the standard Euclidean distance between $v \in \mathbb{R}^{m}$ and $W$. The designation $\omega(v)$ refers to the unique point in $W$ closest to $v$. We call $(t, x) \in[0, T] \times M$ a maximal distance pair if

$$
\operatorname{dist}_{W} f(t, x)=\sup _{y \in M} \operatorname{dist}_{W} f(t, y)>0 .
$$

Let $\lambda(v)$ denote the difference $v-\omega(v)$ for $v \in \mathbb{R}^{m}$.

Following the proof of Theorem 2.1, one can obtain the following result.

Theorem 3.1. Suppose the vector-valued functions $f(t, x)$ and $\phi(t, x, v)$ related by equations (3.1) for $i=1, \ldots, m$ meet the following requirements:

(1) The initial value $f(0, x)$ lies in $W$ for all $x \in M$.

(2) The estimate $\langle\lambda, \phi(t, x, \omega)\rangle \leq 0$ holds for any $(t, x, \omega) \in(0, T) \times M \times \partial W$ and any supporting vector $\lambda \in S_{\omega} W$.

If the value $f(t, x)$ lies outside of $W$ for some $(t, x) \in(0, T] \times M$, then there exists a maximal distance pair $\left(t_{\mathrm{pos}}, x_{\mathrm{pos}}\right) \in(0, T) \times \partial M$ such that the formula

$$
\left.\frac{\partial}{\partial v}\left\langle\lambda\left(f\left(t_{\mathrm{pos}}, x_{\mathrm{pos}}\right)\right), f\left(t_{\mathrm{pos}}, x\right)\right\rangle\right|_{x=x_{\mathrm{pos}}}>0
$$

holds true.

Acknowledgements. I express my profound gratitude to Prof. Leonard Gross for his support and numerous productive discussions.

\section{References}

[1] L. Amour and T. Raoux, The Cauchy problem for a coupled semilinear parabolic system. Nonlinear Anal. 52 (2003), 891-904. Zbl 1015.35037 MR 1937871

[2] H.-D. Cao and L. Ni, Matrix Li-Yau-Hamilton estimates for the heat equation on Kähler manifolds. Math. Ann. 331 (2005), 795-807. Zbl 1083.58024 MR 2148797

[3] B. Chow, The Yamabe flow on locally conformally flat manifolds with positive Ricci curvature. Comm. Pure Appl. Math. 45 (1992), 1003-1014. Zbl 0785.53027 MR 1168117

[4] B. Chow, Ricci flow and Einstein metrics in low dimensions. In Surveys in differential geometry: essays on Einstein manifolds, International Press, Boston 1999, 187-220. Zbl 0999.53041 MR 1798610

[5] B. Chow and D. Knopf, The Ricci flow: an introduction. Math. Surveys Monogr. 110, Amer. Math. Soc., Providence, RI, 2004. Zbl 1086.53085 MR 2061425

[6] B. Chow and P. Lu, The maximum principle for systems of parabolic equations subject to an avoidance set. Pacific J. Math. 214 (2004), 201-222. Zbl 1049.35101 MR 2042930 
Vol. 83 (2008) The Hopf boundary point lemma for vector bundle sections

[7] C. Cosner and P. Schaefer, On the development of functionals which satisfy a maximum principle. Appl. Anal. 26 (1987), 45-60. Zbl 0607.34049 MR 0916898

[8] R. Hamilton, Three-manifolds with positive Ricci curvature. J. Differential Geom. 17 (1982), 255-306. Zbl 0504.53034 MR 0664497

[9] R. Hamilton, Four-manifolds with positive curvature operator. J. Differential Geom. 24 (1986), 153-179. Zbl 0628.53042 MR 0862046

[10] R. Hamilton, A matrix Harnack estimate for the heat equation. Comm. Anal. Geom. 1 (1993), 113-126. Zbl 0799.53048 MR 1230276

[11] H. Kuiper, Invariant sets for nonlinear elliptic and parabolic systems. SIAM J. Math. Anal. 11 (1980), 1075-1103. Zbl 0468.35055 MR 0595833

[12] H. Kuiper, Positively invariant regions for strongly coupled reaction-diffusion systems with a balance law. J. Math. Anal. Appl. 249 (2000), 340-350. Zbl 0984.35084 MR 1781228

[13] R. Redheffer and W. Walter, Invariant sets for systems of partial differential equations I. Parabolic equations. Arch. Rational Mech. Anal. 67 (1978), 41-52. Zbl 0377.35038 MR 0473317

[14] Y. Shen, On Ricci deformation of a Riemannian metric on manifold with boundary. Pacific J. Math. 173 (1996), 203-221. Zbl 0867.53031 MR 1387799

[15] J. Smoller, Shock waves and reaction-diffusion equations. Grundlehren Math. Wiss. 258, 2nd edition, Springer-Verlag, New York 1994. Zbl 0807.35002 MR 1301779

[16] H. Weinberger, Invariant sets for weakly coupled parabolic and elliptic systems. Rend. Mat. (6) 8 (1975), 295-310. Zbl 0312.35043 MR 0397126

Received August 1, 2006

Artem Pulemotov, Department of Mathematics, Cornell University, 310 Malott Hall, Ithaca, NY 14853-4201, U.S.A.

E-mail: artem@math.cornell.edu 